\title{
Genetic algorithm for solving celestial navigation fix problems
}

\author{
Ming-Cheng Tsou, Ph.D. \\ National Kaohsiung Marine University, Taiwan
}

\begin{abstract}
As we enter the $21^{\text {st }}$ century and advance further into the information age, traditional methods for computing a celestial navigation fix can no longer meet the requirements of modern vessels in terms of calculation speed and precision. Study of precise, rapid, and convenient celestial navigation computational methods and the application of information technology to modern celestial navigation is especially meaningful, considering the current push for e-Navigation. In this work, we employ a genetic algorithm, from the field of artificial intelligence, due to its superior search ability that mimics the natural process of biological evolution. Unique encodings and genetic operators designed in this study, in combination with the fix principle of celestial circles of equal altitude in celestial navigation, allow the rapid and direct attainment of accurate optimum vessel position. Test results indicate that this method has more flexibility, and avoids tedious and complicated computation and graphical procedures.
\end{abstract}

Key words: genetic algorithm; celestial navigation; intercept method; celestial fix

\section{INTRODUCTION}

Celestial navigation is one of the important positioning and navigation methods for ocean voyages due to its low cost, high reliability, strong independence, and inability to be detected. It is also one of the traditional navigational skills required of navigators by the International Maritime Organization (IMO). However, since 1994, the revolutionary Global Positioning System (GPS) has become widely available to the general public. GPS meets almost all of the requirements of seafarers, such as high precision, all weather functionality, automatic operation, and global coverage. This has resulted in the successive abandonment of other radio navigation systems (e.g. OMEGA, NNSS, DECCA, and LORAN A). The celestial navigation system, with its longstanding history, also faces a severe situation. However, while we enjoy the convenience that GPS has brought us, we are also exposed to the risks arising from our over-reliance on it. Navigation safety is endangered when satellite signals are cut off or interfered with, or when the satellite receiver system is broken; both situations render positioning of the vessel impossible. Even today, when navigation is dominated by GPS, a celestial fix still serves as an important backup measure. Nonetheless, as we enter the $21^{\text {st }}$ century and advance into the information age, traditional methods for computing a celestial navigation fix can no longer meet the requirements of modern vessels in terms of calculation speed and precision.

At the Manila Conference hosted by the IMO in 2010, a new amendment regarding the Seafarers' Training, Certification, and Watchkeeping (STCW) Code was issued. The amendment describes e-Navigation as the focus of the development of navigation technology, and the application of information technology as a key direction of development. In addition, while celestial navigation received sustained emphasis in the Manila amendment, there is an explicit encouragement for the usage of electronic nautical almanacs and celestial navigation calculation software in Section B-II/1 of Chapter 2, "Guidance regarding the master and the deck department", which concerns training and competency requirements. This can be seen as an effort to lead the development of traditional celestial navigation gradually toward e-Navigation.

For these reasons, the study of high precision, rapid, and convenient celestial navigation computational methods and the application of information technology to modern celestial navigation is very meaningful. This work is based on the concept of the genetic algorithm from the field of artificial intelligence. It combines a search method that mimics the natural process of biological evolution with the celestial fix principle of circles of equal altitude to rapidly and directly obtain accurate optimum vessel position data. Test results show that in addition to avoiding tedious and complicated computation and graphical procedures, this method is also more flexible, and is consistent with the development trend of celestial navigation in the electronic and information age.

\section{DEVELOPMENT OF CONTEMPORARY CELESTIAL NAVIGATION TECHNOLOGY}

The objective of celestial navigation, as traditionally practiced, is to determine the latitude and longitude of a vessel 
at a specific time using observations of the altitudes of celestial bodies. This objective and the basic principles still remain unchanged today, and have formed a foundation on which a number of methods have been developed. The following is a review of the development of contemporary celestial navigation technology.

\section{Intercept Method}

The intercept method, which is the oldest method, was developed in 1875 by Marcq de St. Hilaire. This method translates each celestial altitude observation into a line of position (LOP) on the surface of the Earth. In principle, a series of observations defines a group of intersecting LOPs and this intersection represents the observer's position the fix. Having been practiced for more than a hundred years, the intercept method is able to provide complete solutions for problems of astronomical vessel positioning. Its applicability continues in today's maritime education and training, and in merchant shipping practice. However, it is an approximate method and involves graphical procedures. The entire process consists of observing, calculating, and plotting. Even when performed by professional seafarers, one astronomical positioning will take around 20 minutes. Considering the high speed of modern vessels, this method fails to achieve real-time positioning, and the lag time in positioning cannot guarantee navigation safety. Moreover, there are two theoretical assumptions implied in the intercept method. One is that the azimuth at the observer's position with respect to the celestial body's geographical position is the same as the azimuth at the assumed position. The other assumption is that when the co-altitude is sufficiently large, the circle of position can be taken as line of position. In view of these assumptions, the accuracy of astronomical vessel positioning is subject to the following two restrictions:

1) As the choice of assumed position (AP) is by a trial-anderror method, its distance to the actual vessel position largely affects the accuracy of the result. Therefore, this distance should not exceed 30 NM.

2) The altitude of the celestial body should not exceed 70 degrees. Otherwise, the resulting error of curvature will increase when using line of position in lieu of circle of position on the Mercator Chart due to the reduced coaltitude.

Following the widespread application of information technology, some researchers applied the intercept method using computer programs. In Van Allen [13], the development is based on the geometry of the two LOPs. A least-squares approach for a multi-star fix is presented in Dewit [4] based on the plane geometry and straight lines formed by LOPs near the estimated position. This method is a direct mathematical translation of chart-based navigation. Although the intercept method has been computerized, the inherent drawbacks due to the two aforementioned assumptions still exist and limit its accuracy.

\section{Spherical Triangle Method (STM) and Vector-Matrix Method}

With the aid of computer programs, problems that could not be solved previously using the inspection table can now be solved using the STM or Vector-Matrix methods. With these tools, the celestial fix becomes fast and accurate. Unlike with the intercept method, there is no limitation on accuracy. Either the assumed position is not needed or the knowledge of an approximate position is sufficient. Some researchers have studied a two-body fix $[2,3,5,6,12,14]$. It is proposed that at a given time, the astronomical vessel position can be determined by observing circles of equal altitude of two celestial bodies. In a multi-body celestial fix, a statistical method such as the least mean squares technique is applied on the results of a number of two-body fixes to deal with the over-determined celestial fix problem and to solve for the final position [1]. However, in practice, it is rare to use two celestial bodies to obtain a vessel fix, and the statistical computation adds complexity to this method. Some other researchers have studied the multi-body celestial fix by invoking the circle of equal altitude. In this method, a celestial body's altitude is viewed as a function of latitude and longitude, and a multi-body celestial fix is carried out directly, using the vector-matrix method, instead of indirectly using results of a two-body fix $[9,11,15]$. The vectormatrix formulations, while elegant, have the disadvantage that the solution still assumes prior knowledge of the observer's position. Furthermore, the algorithm that proceeds from some initial value by sliding down the gradient of the goodness-offit parameter can converge to a local minimum that is not the best solution [7]. In addition, a multi-body celestial fix that is based on the least squares fit requires more than three celestial bodies. When there are only two celestial bodies available, this method cannot be used. In these cases, a virtual celestial body is introduced according to the vessel's dead reckoning position. Consequently, the accuracy is affected by the assumed vessel position.

\section{GENETIC ALGORITHM TO SOLVE FOR ASTRONOMICAL VESSEL POSITION}

Advances in information technology have brought new changes to celestial navigation, especially in the early 1990s when research in this field blossomed. At this time, artificial intelligence began to be widely used in various research fields. Unfortunately, research on celestial navigation was soon largely replaced by the rapid emergence and development of GPS. At present, celestial navigation still serves as an important backup measure of vessel positioning in the ocean. It is therefore necessary to investigate the applications of information technology and artificial intelligence technology in celestial navigation and to improve the shortcomings of existing methods, which were described in the previous section.

\section{General principle of $G A$}

In recent years, the development of artificial intelligence technology has enjoyed mutual influence with the life sciences. The flourishing of evolutionary computation methods, such as the genetic algorithm (GA), reflects this characteristic and development trend. GAs are among the most effective methods for optimization problems. All types of objective functions and constraints can be processed without many mathematical prerequisites. During the search process, knowledge of the search space can be automatically obtained and accumulated. Accordingly, the search is modified to solve for the optimal solution or a less suboptimal solution. This method is very efficient and enables a parallel search. Two main features of GAs are a search strategy in a population and the exchange of information between individuals in a population. It simulates the overall learning process involved in the creation of a population from individuals. Starting from an initial population, in which each individual is a candidate solution in the search space of an optimization problem, the fitness of each individual is evaluated based on a fitness function. Multiple individuals with better fitness are subsequently selected, and then modified through 
genetic manipulations such as crossover (analogous to the exchange of information between individuals in nature) and mutation (where an individual is subjected to a sudden change and escapes the local optimum) to form a new population, thus achieving screening and evolution. The process is repeatedly executed. During the iteration process, every new population is superior to the population in the previous generation. Individuals continuously evolve toward better solutions, and eventually reach the optimum solution.

There is always complexity and uncertainty associated with a celestial fix, which makes it difficult to obtain the exact solution mathematically. In this study, the superior search ability of GA is combined with fundamental positioning principles in celestial navigation to perform a fitting calculation for the optimum vessel position.

\section{The celestial fix principle of circles of equal altitude}

The central idea behind the celestial circle-of-equal-altitude fix is to find the best fit to the altitude of a celestial body observed as a function of time. The calculation altitude (Hc) of a celestial body is given as a function of the declination of body (Dec), the Greenwich hour angle (GHA), the observer's assumed longitude $(\lambda)$, and the observer's assumed latitude (L) by:

$$
\begin{gathered}
\operatorname{Sin} \mathrm{Hc}_{\mathrm{i}}=\operatorname{SinL} \times \operatorname{SinDec}_{\mathrm{i}}+ \\
+\operatorname{Cos} \mathrm{L} \times \operatorname{CosDec}_{\mathrm{i}} \times \operatorname{Cos}\left(\mathrm{GHA}_{\mathrm{i}}-\lambda\right)
\end{gathered}
$$

where $i=1$ to $n$ represents the celestial body and the observation data of the $i^{\text {th }}$ celestial body

The GHA and the Dec can be found from the time of observation using the Nautical Almanac or the electronic edition of Nautical Almanac. Hc in Equation 1 is the calculated altitude, which differs from the actual observed altitude. The altitude of a celestial body (hs) is measured by a sextant. An instrumental error correction (I), index correction (IC), and correction for optical deviations such as dip and refractions are applied to the sextant altitude. The resulting value is the actual observed altitude (Ho). Since the actual vessel position can be seen as a function of the altitude of the celestial body, which is a nonlinear function of $\mathrm{L}$ and $\lambda$, we cannot solve equation 1 directly. Therefore, the latitude and longitude of the actual vessel position may be obtained from the difference between Hc and Ho using an appropriate fitting algorithm. With the appropriate Dec and GHA in equation 1, any combination of altitudes of one or several celestial bodies can be used, and no plots or tables are required. Furthermore, when performing the multi-body celestial fix, different observing times lead to inconsistency in the observed altitude. It is therefore necessary to make corrections on the running fix. In this study, the correction approach proposed by Kaplan [8] is adopted.

\section{Implementation of a GA for the celestial fix problem}

The optimum vessel position in a majority of multi-body celestial fix problems is found by data fitting using the least mean squares method, and it is classified as an optimization problem. However, there still exists the problem of converging to a local minimum. GA shows excellent performance in optimum fix processing problems. Its main advantages include: (a) simple calculation without any complicated mathematical procedures; (b) it does not suffer from possible sensitivity to the initial position chosen to start the iterative process, meaning it has less of a tendency to converge toward the local optimum; (c) more flexibility in practice and rapid convergence toward the final result. The GA approach can be used to obtain a fix from multiple celestial observations of a single celestial body or of multiple celestial bodies (more observation data points produce better results). This method is in line with the needs of modern navigation. The procedure includes:

1) Design of the objective function: The first step in solving an optimization problem is the design of the objective function to evaluate the fitness of each individual. A GA is used in this study to minimize the root mean squares of the altitude residuals (equation 2, root mean square error - RMSE), defined as the difference between each observed altitude (Ho) and the altitude computed from equation 1 (Hc). The residual defined differs from the altitude difference normally used to plot LOPs since the altitude difference is referred to an assumed position chosen to permit table lookup of whole degrees of Local Hour Angle. When the RMSE converges to meet the acceptance criteria, the optimum vessel position is obtained.

$$
\text { RMSE }=\min \sqrt{\frac{\sum_{i=1}^{n}\left(\mathrm{Ho}_{\mathrm{i}}-\mathrm{Hc}_{\mathrm{i}}\right)^{2}}{\mathrm{n}}}
$$

2) Design and encoding of individuals and population: In order to improve the efficiency and accuracy in this study, individual encodings in the GA are not in the form of traditional binary strings. Instead, real number encodings are adopted. Each individual contains two real number columns, representing latitude and longitude, to represent a possible solution (vessel position). Each population consists of multiple individuals. In generating the initial population, a reference position is introduced to improve search efficiency. This reference position is different from the assumed position in the intercept method. It is an approximate position of reference, which is not required to be within $30 \mathrm{NM}$ of the actual vessel position; indeed, it can be hundreds of nautical miles away. This permits a large degree of flexibility in choosing the reference position. This position is mainly a reference for a heuristic search. However, it is certain that a reference position closer to the actual position results in a smaller search space, resulting in higher efficiency and a better quality solution. The introduction of a new reference position does not add much inconvenience to seafarers. As stated by Pepperday [10], "If you know your approximate position. Why not use it?". In fact, methods that claim that no DR reference position is required still need to assume prior knowledge of the observer's position, or that it be obtained through complicated computational procedures. Moreover, DR position is also required for applying corrections on the running fix. The reference position is used as the datum point in this work. In the user defined maximum search degree and numbers of generations, random numbers are used to generate the initial position data of each individual in the initial population as the starting point of the evolution (equation 3, 4, 5):

Data structure of each individual Di: $\left\{\begin{array}{l}\text { double latitude } \\ \text { double longitude }\end{array}\right.$

Data of every initial individual $\left\{\begin{array}{l}\text { IniL }+ \text { Tol } \times \text { RandNum } \\ \text { Ini } \lambda+\text { Tol } \times \text { RandNum }\end{array}\right.$ 


$$
\text { Initial Population }\left(\mathrm{P}_{1}\right)=\left\{\mathrm{D}_{11}, \mathrm{D}_{12}, \mathrm{D}_{13}, \mathrm{D}_{14}, \ldots, \mathrm{D}_{1 \mathrm{n}}\right\}
$$

where IniL and Ini $\lambda$ represent the latitude and longitude values of the reference position, respectively. Tol is the user defined maximum search degree. RandNum is random real number between -1 and +1 , and the random positions are generated to correct for the reference position. $\mathrm{P}_{1}$ is the initial population, and $\mathrm{n}$ is the number of individuals in a population.

3) Selection: The traditional roulette method is adopted as the selection method. This is a proportional selection strategy. In this method, individuals with a higher fitness level have a better chance of being selected to generate the new population. This concept is used in GA to mimic biological evolution.

4) Crossover: The crossover rate is denoted as $P_{c} \cdot n$ individuals in the population are stochastically paired to form $n / 2$ pairs. In order to determine in which pairs crossover takes place, the following procedure is repeated from $\mathrm{i}=1$ to $\mathrm{n} / 2$ : Generate a random number $\mathrm{r}$ in the interval $[0,1]$, and if $r<\mathrm{P}_{c}$, then apply crossover on the $\mathrm{i}^{\text {th }}$ pair. The crossover proceeds according to equation 6 and 7 :

$$
\mathrm{D}_{\mathrm{New}}:\left\{\begin{array}{l}
\mathrm{L}_{\mathrm{New}}=\mathrm{L}_{1}+\left|\mathrm{L}_{1}-\mathrm{L}_{2}\right| \times \text { RandNum } \\
\lambda_{\mathrm{New}}=\lambda_{1}+\left|\lambda_{1}-\lambda_{2}\right| \times \text { RandNum }
\end{array}\right.
$$

or:

$$
\mathrm{D}_{\mathrm{New}}:\left\{\begin{array}{l}
\mathrm{L}_{\mathrm{New}}=\mathrm{L}_{2}+\left|\mathrm{L}_{1}-\mathrm{L}_{2}\right| \times \text { RandNum } \\
\lambda_{\mathrm{New}}=\lambda_{2}+\left|\lambda_{1}-\lambda_{2}\right| \times \text { RandNum }
\end{array}\right.
$$

where $D_{\text {New }}$ represents new individuals generated through crossover. $\mathrm{L}_{\mathrm{New}}$ and $\lambda_{\mathrm{New}}$ denotes the latitude and longitude of new individuals generated through cross. $\mathrm{L}_{1}, \lambda_{1}, \mathrm{~L}_{2}, \lambda_{2}$ are latitude and longitude of the parent individuals on which crossover is applied. RandNum is random real number between -1 and +1 , and they are used to generate more optimum random positions. The crossover step can bring new positions closer (as shown in Figure 1) or further away (as shown in Figure 2). Since the parent individuals are already individuals with better fitness, it is more likely for the newly generated individuals to have further improvement (more closer to the actual vessel position).

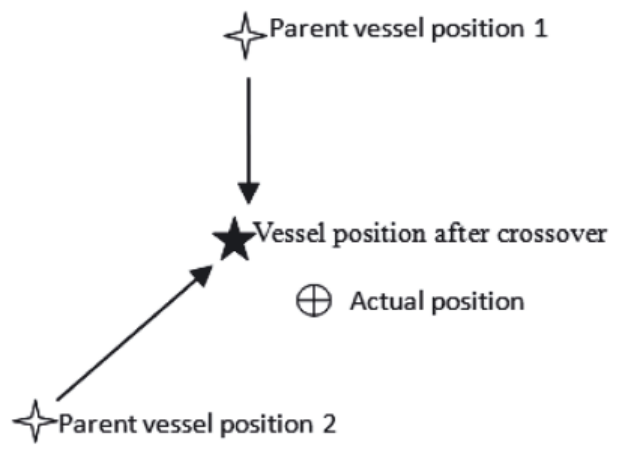

Fig. 1. Position becomes closer after crossover

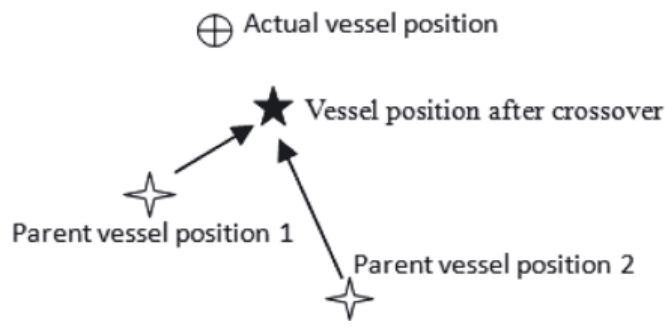

Fig. 2. Position becomes further away after crossover
5) Mutation: The mutation rate is defined as $P_{m}$ The following procedure is repeated on individual $i=1$ to $n$ : Generate a random number $\mathrm{r}$ in the interval $[0,1]$, and if $\mathrm{r}<\mathrm{P}_{\mathrm{m}}$ then apply the mutation operation according to:

$$
\mathrm{D}_{\text {Mutation }}:\left\{\begin{array}{c}
\mathrm{L}_{\text {Mutation }}=\mathrm{L}_{\text {Old }}+\text { Noise } \times \text { RandNum } \\
\quad \text { or } \\
\lambda_{\text {Mutation }}=\lambda_{\text {Old }}+\text { Noise } \times \text { RandNum }
\end{array}\right.
$$

$\mathrm{D}_{\text {Mutation }}$ represents the data of individuals after mutation. $\mathrm{L}_{\text {Mutation }}$ and $\lambda_{\text {Mutation }}$ represent the latitude and longitude of individuals after mutation. $\mathrm{L}_{\text {Old }}$ and $\lambda_{\text {Old }}$ represent the latitude and longitude of individuals before the mutation. Noise is a user defined degree of allowable perturbation, in place for starting new research. RandNum is random real number between -1 and +1 , and these numbers are used to generate the stochastic perturbation degree. The mutation operation can prevent the search from converging to the local optimum (as shown in Figure 3).

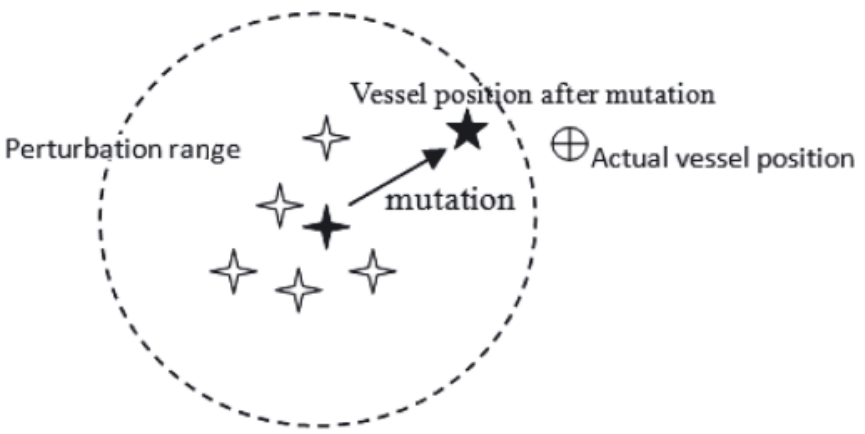

Fig. 3. Mutation prevents the search from converging to the local optimum

6) Improvement of the efficiency of the GA: In some cases, individuals in the population become increasingly similar to each other during the evolutionary process, thus limiting the GA's global search ability, and causing the GA to converge toward a local optimum instead of the global optimum. To avoid this, we have enhanced the global search ability of the GA in the current study. At the early stage of evolution, a large crossover rate and mutation rate are imposed in order to maintain diversity in the population. At the later stages of evolution, individuals have already developed to a near optimal state. At this time, a large crossover rate or large mutation rate are likely to impair individual fitness. Therefore, the crossover rate and mutation rate are reduced at the later stages.

\section{Procedures of implementation}

Although a large population size is helpful in the search of vessel position, it inevitably increases the search time, making it difficult to use for real time positioning. In the current study, the population size is set at 50 individuals. The crossover rate and mutation rate are 0.6 and 0.05 , respectively. The maximum search space is set to 2 degrees (i.e. 4 degrees in latitude and longitude), which is sufficient for navigation. The perturbation range is set to 1 degree. Applying these settings in the genetic operations creates new generations to replace the parent generation. The search is terminated when either the RMSE converges to the acceptance criteria, or a fixed number of generations is reached. Termination of the search indicates that the optimal vessel position has been obtained. Experiments show that convergence to the accurate position generally occurs within 50 generations, and within approximately 5 seconds. 


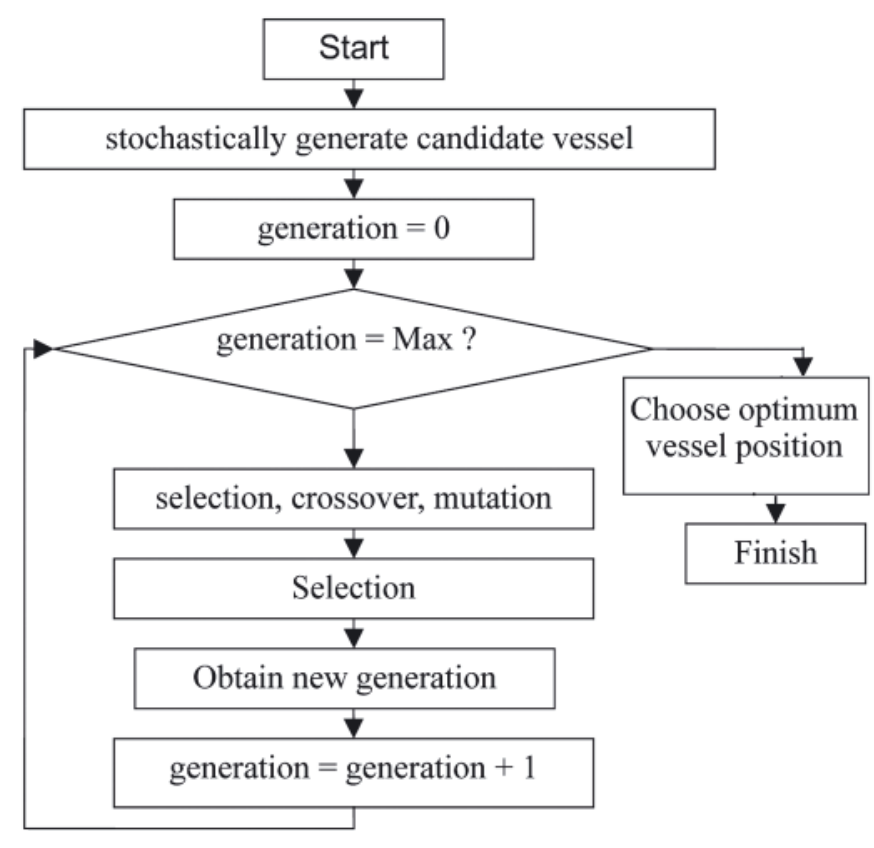

Fig. 4. The flowchart of GA for obtaining the optimum vessel position

This satisfies the requirement of real-time positioning. The step-by-step procedures are (refer to Figure 4):

1) Use real numbers to encode individuals in the solution space. Each individual corresponds to a candidate vessel position.

2) Set the initial population size to contain 50 individuals, i.e. stochastically generate 50 candidate vessel positions.
3) Calculate the value of the fitness function of each individual according to equation (1) and (2), and determine which individuals in the population are relatively close to the actual vessel position.

4) Select individuals close to the actual vessel positions using the roulette technique.

5) Apply crossover to the population. Crossover between vessel positions with good fitness generates vessel positions that are closer to the optimal positions.

6) Apply genetic mutation to the population to avoid converging to local optima instead of the global optimum.

7) Re-calculate the value of the fitness function of each individual according to equations (1) and (2).

8) Determine whether the termination criteria has been met. If yes, then the genetic operation ends and proceeds to step (9), where the current optimum vessel position is exported. Otherwise, move back to step (4).

9) Export the optimum vessel position.

\section{RESULT VALIDATION}

The validity of this study is verified by comparing results of a two celestial body fix and a multiple celestial body fix with those obtained by other methods. Visual Basic.Net 2010 is used as the development tool. In order to add visual effects, components of Geographic Information System (GIS) are also introduced. This is also aimed at future celestial navigation education and integration with an Electronic Chart Display and Information System (ECDIS).

Tab. 1. Comparison of GA and relevant information from Hsu et al. [6] for two-body fix

\begin{tabular}{|c|c|c|c|c|}
\hline Body & ZT & Ho & GHA & Dec \\
\hline Capella & $20-03-58$ & $15^{\circ} 19.3^{\prime}$ & $131^{\circ} 24.8^{\prime}$ & $45^{\circ} 58.4^{\prime} \mathrm{N}$ \\
\hline Alkaid & $20-02-56$ & $77^{\circ} 34.9^{\prime}$ & $003^{\circ} 14.2^{\prime}$ & $49^{\circ} 25.7^{\prime} \mathrm{N}$ \\
\hline DR (20-04-00) & & $\mathrm{L}=41^{\circ} 34.8^{\prime} \mathrm{N}$ & $\lambda=017^{\circ} 00.5^{\prime} \mathrm{W}$ & \\
\hline Intercept Method & $\mathrm{L}=41^{\circ} 38.6^{\prime} \mathrm{N} \quad \lambda=017^{\circ} 08.1^{\prime} \mathrm{W}$ \\
\hline SEEM & $\mathrm{L}=41^{\circ} 39.1^{\prime} \mathrm{N}$ & $\lambda=017^{\circ} 07.3^{\prime} \mathrm{W}$ \\
\hline GA & $\mathrm{L}=41^{\circ} 39.1^{\prime} \mathrm{N}$ & $\lambda=017^{\circ} 07.3^{\prime} \mathrm{W}$ \\
\hline
\end{tabular}

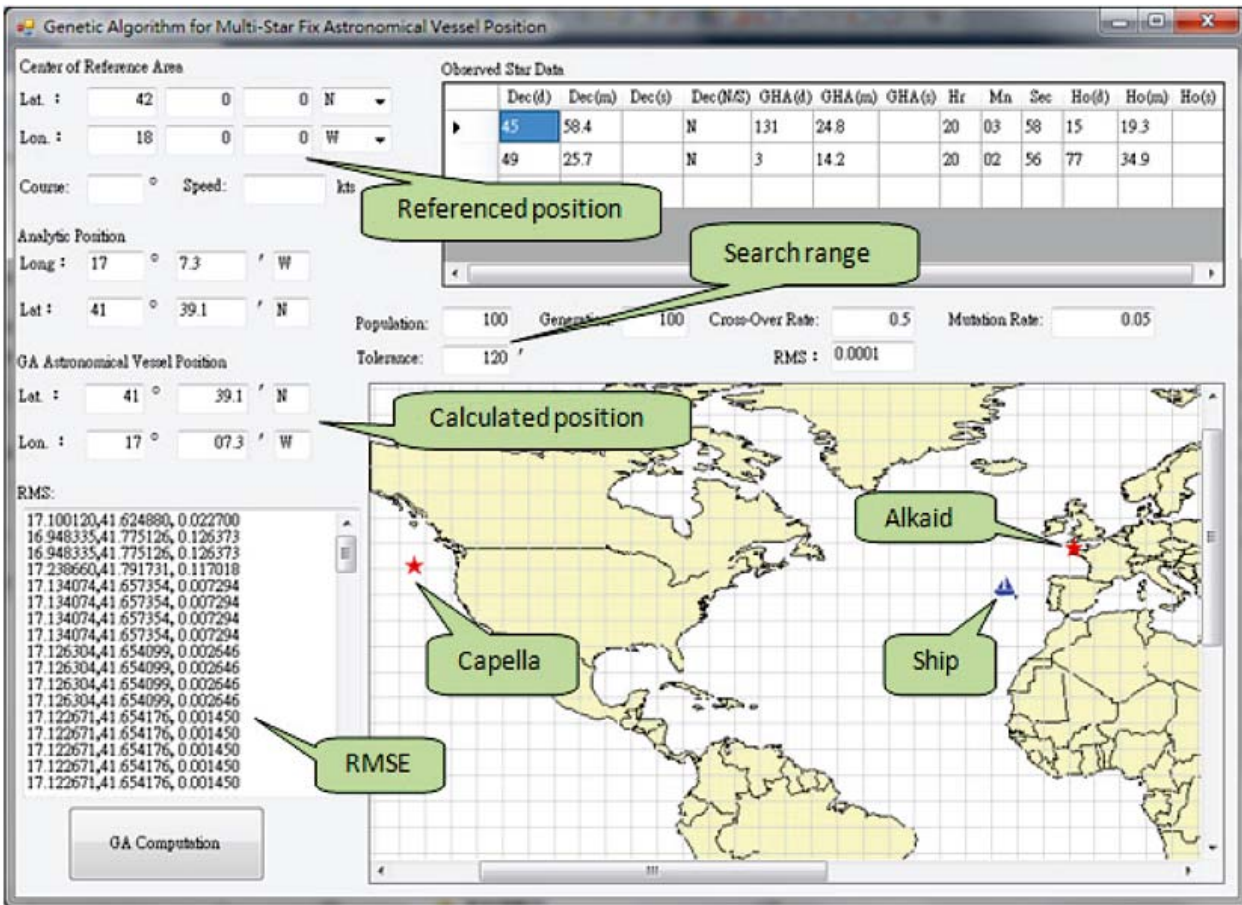

Fig. 5. GA of two-body celestial fix 


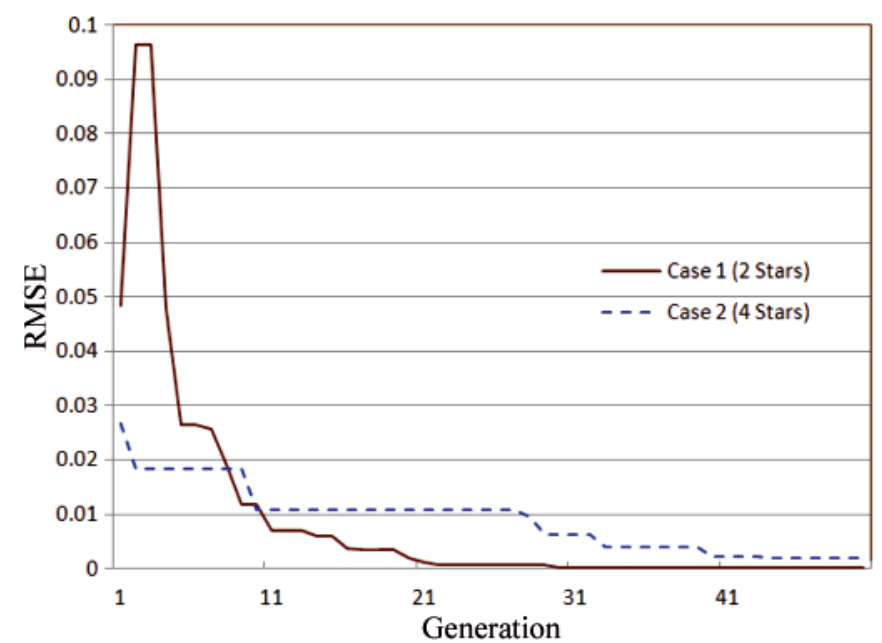

Fig. 6. The evolutionary process for calculation of a fix

Case 1 - Two-Body Fix: The data in this case study is taken from Hsu et al. [6]. The authors used the Simultaneous Equalaltitude Equation Method (SEEM) to perform a two-body celestial fix. Table 1 contains relevant experiment data.

It can be seen from Table 1 that the observed altitude of Alkaid is as high as $77^{\circ} 34.9^{\prime}$, which exceeds the upper limit of $70^{\circ}$ of the intercept method. Hsu et al. [6] found that the LOP drawn using the intercept method shifted due to curvature error, thus resulting in an inaccurate vessel position estimate.
The present study however is free from this drawback. In our experiments, even if the distance between the reference position and the actual vessel position exceeds 30NM and the search degree reaches $4^{\circ} \times 4^{\circ}$ (refer to Figure 5), the correct vessel position can still be obtained. Moreover, the RMSE converges to 0.0001 within 30 generations and within 3 seconds (refer to Case 1 in Figure 6). The calculation is performed in a rapid and accurate manner without the need to construct a visual celestial body.

Case 2 - Multi-body Fix: There are four celestial bodies involved in this case study and more realistic in real marine navigation situation. Table 2 presents relevant observational data. In addition to the increased number of celestial bodies, correction on the running fix is also applied. The parameters in this experiment are in general identical to those in the previous case. However, the reference position is deliberately placed further so that it is almost 2 degrees away from the actual vessel position. Though the final RMSE (about 0.002) is slightly bigger than Case 1, caused by observation errors from multi-star sights, it can converge within 50 generations (refer to Case 2 in Figure 6). Results of this experiment show a good agreement with those obtained by the vector-matrix method or the graphical intercept method (refer to Figure 7 and Figure 8). No plots or sight reduction tables are needed. The running time is within 5 seconds, and can meet the demands of real-time navigation fix. The applicability of the GA approach for both the two-body fix and multi-body fix is thus verified.

Tab. 2. Multi-body fix

\begin{tabular}{|c|c|c|c|c|}
\hline Body & ZT (1993/9/13) & Ho & GHA & Dec \\
\hline Altair & $18-00-00$ & $37^{\circ} 53.0^{\prime}$ & $325^{\circ} 06.6^{\prime}$ & $08^{\circ} 51.4^{\prime} \mathrm{N}$ \\
\hline Fomalhaut & $18-04-00$ & $27^{\circ} 54.0^{\prime}$ & $279^{\circ} 24.2^{\prime}$ & $29^{\circ} 39.1^{\prime} \mathrm{S}$ \\
\hline Achernar & $18-08-00$ & $17^{\circ} 46.5^{\prime}$ & $240^{\circ} 21.7^{\prime}$ & $57^{\circ} 15.8^{\prime} \mathrm{S}$ \\
\hline Rasalhague & $18-12-00$ & $41^{\circ} 35.5^{\prime}$ & $002^{\circ} 04.8^{\prime}$ & $12^{\circ} 34.1^{\prime} \mathrm{N}$ \\
\hline DR & $\mathrm{L}=35^{\circ} 13.0^{\prime} \mathrm{S} \lambda=005^{\circ} 20.0^{\prime} \mathrm{E} \quad \mathrm{Course}^{\prime} 220^{\circ} \mathrm{Speed}=18 \mathrm{kts}$ \\
\hline Intercept Method & \multicolumn{3}{|c|}{$\mathrm{L}=35^{\circ} 19.0^{\prime} \mathrm{S} \quad \lambda=005^{\circ} 26.5^{\prime} \mathrm{E}$} \\
\hline Vector-Matrix & $\mathrm{L}=35^{\circ} 18.6^{\prime} \mathrm{S} \quad \lambda=005^{\circ} 26.9^{\prime} \mathrm{E}$ \\
\hline GA & $\mathrm{L}=35^{\circ} 18.6^{\prime} \mathrm{S} \quad \lambda=005^{\circ} 27.0^{\prime} \mathrm{E}$ \\
\hline
\end{tabular}

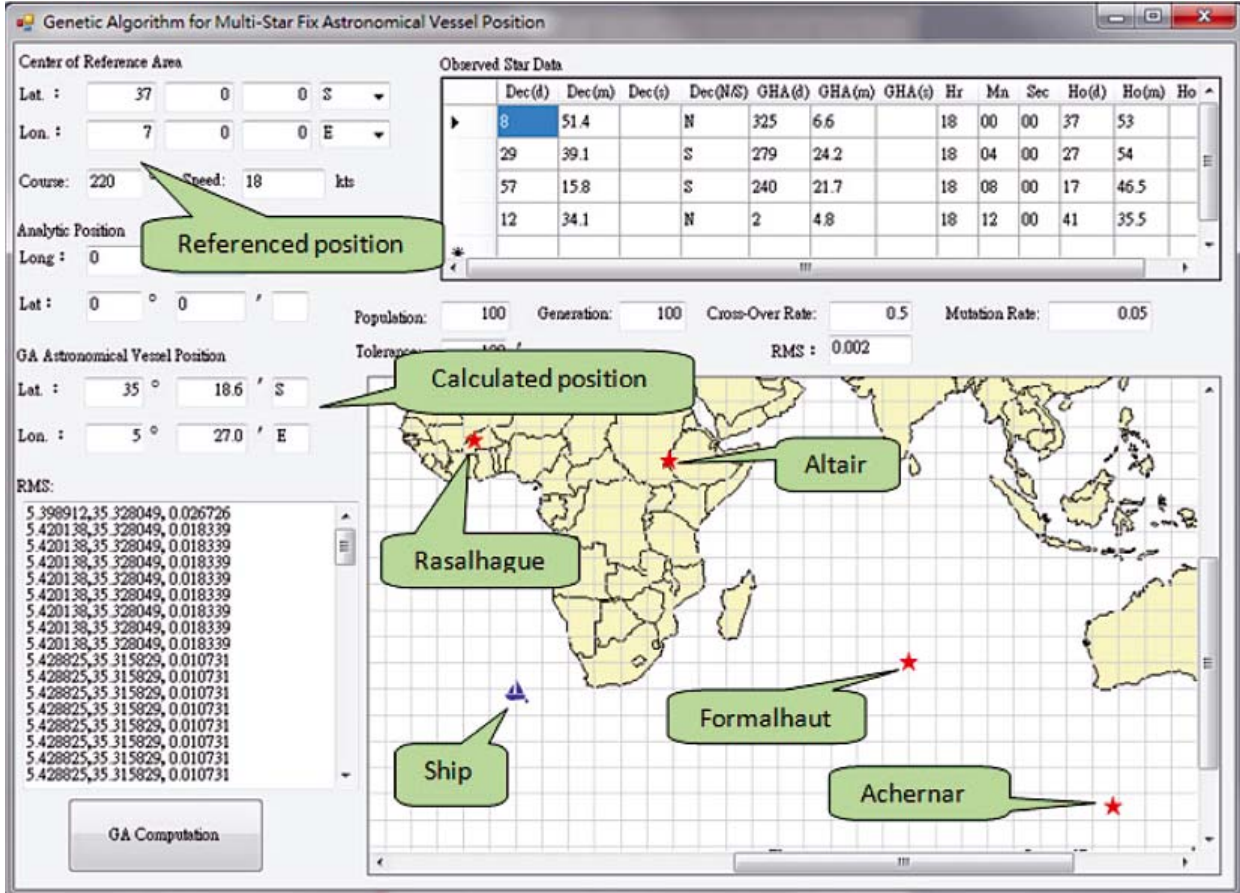

Fig. 7. GA of multi-body celestial fix 


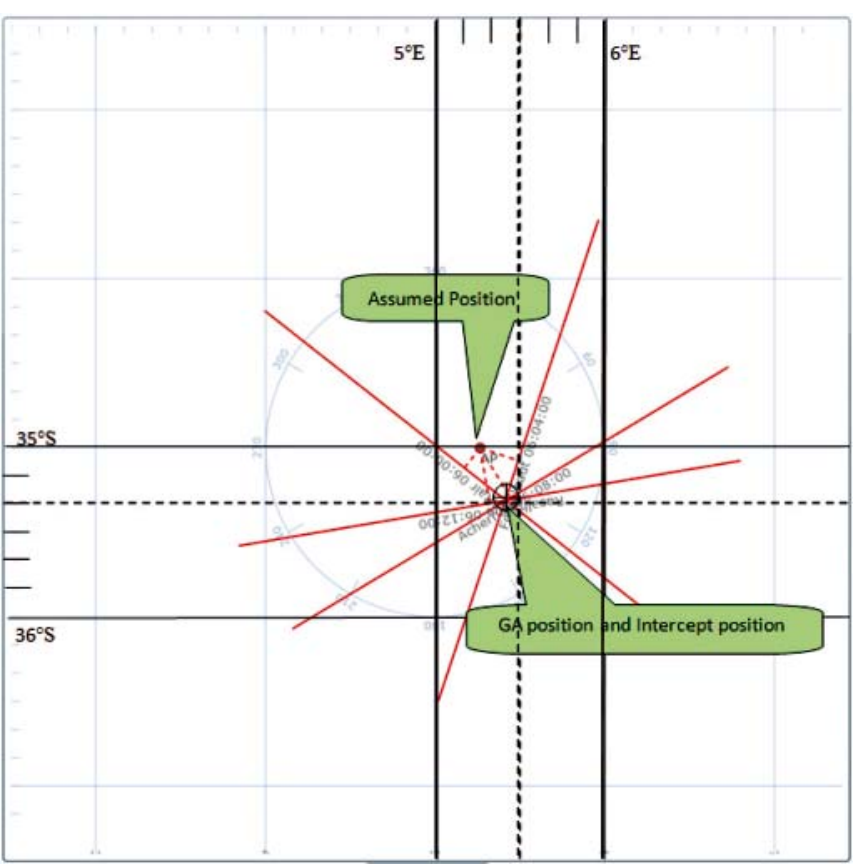

Fig. 8. Comparison of $G A$ and intercept method in multi-body celestial fix

\section{CONCLUSIONS}

Although celestial navigation has its shortcomings, it cannot be replaced by GPS due to the advantages of low cost, high reliability, strong independence, and inability to be detected. In this study, a genetic algorithm that mimics biological evolution, taken from the field of artificial intelligence, is applied to the celestial navigation fix problem. The main features of the GA include: (1) simple calculation without any complicated mathematical procedures; (2) Avoidance of converging toward a local optima; (3) more flexibility in practice and rapid convergence toward the final position estimate. The algorithm can be used to obtain a fix from observation of a single celestial body (multiple observations) or of multiple celestial bodies, and more observation data produces better results. Experimental results show that in addition to evading tedious and complicated computation and graphical procedures, the GA approach is more flexible compared with other methods. This approach also has the potential to be integrated with ECDIS. Moreover, it can also be used to confirm whether GPS is functioning normally. Navigation technology is moving into the age of e-Navigation, and the continuous development of celestial navigation can only be realized through integration with information technology.

\section{BIBLIOGRAPHY}

1. Chen C.-L., Chang J.-R., Hsu T.-P.: A New Computation Method to Solve the LOP Without the Intercept (In Chinese). Maritime Research Journal, 2003, 15, 77-93.

2. Chen C.-L., Hsu T.-P., Chang J.-R.: A Novel Approach to Determine the Astronomical Vessel Position. Journal of Marine Science and Technology, 2003, 11, 221-235.

3. Chiesa A., Chiesa R.: A Mathematical Method of Obtaining an Astronomical Vessel Position. The Journal of Navigation, 1990, 43, 125-129.

4. Dewit C.: Optimal Estimation of a Multi-Star Fix. NAVIGATION, Journal of The Institute of Navigation, 1974, 21, 320-325.

5. Gibson, K.: On the Two-Body Running Fix. The Journal of Navigation, 47, 103-107, 1994.

6. Hsu T.-P., Chen C.-L., Chang J.-R.: New Computation Methods for Solving Problems of the Astronomical Vessel Position. The Journal of Navigation, 2005, 58, 315-335.

7. Kaplan G. H.: Determining the Position and Motion of a Vessel from Celestial Observations. NAVIGATION, Journal of The Institute of Navigation, 1996, 42, 631-649.

8. Kaplan G. H.: The Motion of the Observer in Celestial Navigation, Online Information for the Defense Community, 1996, (Available online at http://www.dtic.mil/cgi-bin/ GetTRDoc Location $=$ U2\&doc $=$ GetTRDoc.pdf\&AD $=$ ADA423226)

9. Metcalf T. R., Metcalf, F. T.: On the Overdetermined Celestial Fix. NAVIGATION, Journal of The Institute of Navigation, 1991, 38, 79-89.

10.Pepperday M.: The 'Two-Body Problem'At Sea. The Journal of Navigation, 1992, 45, 138-142.

11. Severance R. W.: Overdetermined Celestial Fix by Iteration. NAVIGATION, Journal of The Institute of Navigation, 1989, 36, 373-378

12.Spencer B.: Astronomical Fixes Without an Assumed Position. The Journal of Navigation, 1990, 43, 449-451.

13.Van Allen, J. A: An Analytical Solution of the Two Star Sight Problem of Celestial Navigation. Journal of The Institute of Navigation, 1981, 28, 40-43.

14.Vulfovich B., Fogile V.: New Ideas for Celestial Navigation in the Third Millennium. The Journal of Navigation, 2010, 63, 373378

15.Wu G.: An Optimal Estimating Method for Celestial Navigation. The Journal of Navigation, 1991, 44, 266-269.

\section{CONTACT WITH THE AUTHOR}

Ming-Cheng Tsou, Ph.D.

National Kaohsiung Marine University, Taiwan e-mail: d86228006@yahoo.com.tw 